\title{
Development and calibration of a particulate matter measurement device with wireless sensor network function
}

\author{
Duckshin Park*, Soon-Bark Kwon, Youngmin Cho \\ Eco-Transport Research Division, Korea Railroad Research Institute, Uiwang-si, Gyeonggi-do, Korea \\ Email address: \\ dspark@krri.re.kr (D. Park)
}

To cite this article:

Duckshin Park, Soon-Bark Kwon, Youngmin Cho. Development and Calibration of a Particulate Matter Measurement Device with Wireless Sensor Network Function, International Journal of Environmental Monitoring and Analysis. Vol. 1, No. 1, 2013, pp. 15-20. doi: $10.11648 /$ j.ijema.20130101.12

\begin{abstract}
A Zigbee-based ubiquitous sensor network (USN) has many industrial applications and provides flexible measuring environments. In particular, the USN system can replace existing measuring devices in harsh environments such as subway stations. To monitor the intensities of various pollutants and air qualities in subway tunnels, this study applied the USN technique. A novel wireless sensor module, PMX, was designed and manufactured to simultaneously detect PM $_{10}$ and $\mathrm{PM}_{2.5}$. Measurements were conducted at a subway station in Seoul. The PM concentrations using PMX were measured, analyzed, and compared with those obtained using an established commercial dust spectrometer (Grimm Aerosol Technik, 1.109). The measurements were performed from 24 March 2010 to 9 April 2010. PMX and the dust spectrometer measured $\mathrm{PM}_{10}$ levels of 98.3 and $40.7 \mu \mathrm{g} / \mathrm{m}^{3}$, respectively, and $\mathrm{PM}_{2.5}$ concentrations of 86.5 and $16.6 \mu \mathrm{g} / \mathrm{m}^{3}$, respectively. The monitored PM levels were investigated in a bimodal form during the sampling period. The $\mathrm{PM}_{10}$ and $\mathrm{PM}_{2.5}$ average correlations between PMX and the dust spectrometer were $\mathrm{r}^{2}=0.81$ and $\mathrm{r}^{2}=0.97$, respectively. The two systems showed a similar time series trend, even though the measured values differed. A simple correlation analysis of the two data groups showed coefficients of determination of 0.7 for $\mathrm{PM}_{10}$ and 0.9 for $\mathrm{PM}_{2.5}$. The PMX data were mostly concentrated around the trend curve. Therefore, calibration of PMX data was required prior to use in the field. For the calibration, simple linear regression and nonlinear regression were used. The resulting correlation coefficients of simple linear regressions were 0.8 for $\mathrm{PM}_{10}$ and 0.9 for $\mathrm{PM}_{2.5}$, whereas those for nonlinear regressions were 0.7 for $\mathrm{PM}_{10}$ and 0.9 for $\mathrm{PM}_{2.5}$. The higher correlation coefficient for $\mathrm{PM}_{10}$ by the nonlinear regression indicates that it is the better method for calibrating the system developed in this study.
\end{abstract}

Keywords: Particulate Matter, Ubiquitous Sensor Network, Subway, Indoor Air Quality

\section{Introduction}

Previous studies have reported the negative effects of fine dust on human health. For example, daily death rates were found to increase by $0.5 \sim 1.5 \%$ when the fine dust concentration in the atmosphere increased (Pope, 1995), and incidences of disease also increased as the fine dust concentration increased (Dockery et al., 1993).

Measurement of fine dust both indoors and outdoors can be performed by a mass concentration method using a filter, a beta ray absorption method using beta rays, and a light scattering method based on the light scattering of dust. Although the mass concentration method is ideal for studies requiring chemical composition, it has a low time-series resolution and requires an extended period of time for data collection. Furthermore, it can both under-measure (due to volatile substances such as ammonium nitrate and organic carbon) and over-measure (due to gas condensation) the dust mass (Chow, 1998).

The light scattering method, which determines the concentration of fine dust based on the size, shape, and refractive index, has high measurement error in terms of dust concentration and size because of humidity. However, it is still used in various studies because it enables continuous measurement and is simple to conduct (Coffey, 2010).

Recently, subway operators in South Korea have been 
attempting to reduce the fine dust concentration in subway stations. The most common way to reduce fine dust is to dilute inside air through ventilation using continuously running large-capacity ventilation fans. The portion of HVAC operating costs within total operating costs is not precisely known, but it is estimated that fan operation accounts for most of the cost. Because HVAC systems dedicated to fine dust concentration are not commonly used, it would be very useful to develop an accurate and low-cost fine dust measurement system.

In this study, we developed a dust measurement system that can monitor fine dust in subway stations or large indoor areas at a lower cost than existing equipment. The novel product features real-time fine dust monitoring and wireless sensor network functions. For evaluation, the developed system and an existing commercial product were installed in the same place, and the developed system was calibrated using various statistical analyses. This study provides the reference data required for calibration when a new fine dust measurement system is developed to replace existing commercial products.

\section{Materials and Methods}

The fine dust measurement system (PMX) developed in this study features a radio frequency (RF) amplifier mounted onto a Zigbee, and uses the wireless sensor network to transfer data up to $1 \mathrm{~km}$ wirelessly in clear space. The fine dust measurement range of PMX is $0 \sim 10,000 \mu \mathrm{g} / \mathrm{m}^{3}$ (grain density $=1.0$ basis), the flow rate is $2 \mathrm{l} / \mathrm{min}$, and the system has an eight-channel $(0.3,0.5,1.0$, $1.5,2.5,5,7,10 \mu \mathrm{m})$ size range. In addition to fine dust, the PMX can include built-in $\mathrm{CO}, \mathrm{CO}_{2}$, temperature, and humidity sensors to simultaneously measure different indoor air qualities.

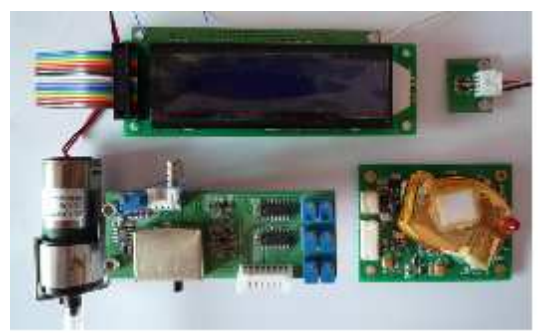

a) Interior of PMX

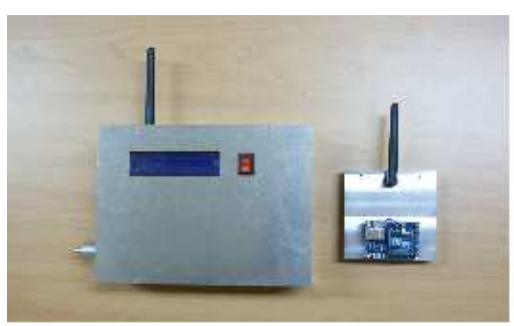

b) Exterior of PMX

Fig. 1. View of the wireless PMX sampler.
The PMX was installed in the entrance to a tunnel located about $200 \mathrm{~m}$ from Gireum Station toward the Miasamgeori Station of Seoul Metropolitan Subway Line No. 4. Fine dust was measured for about 1 month beginning on 25 March 2010. For comparison with PMX, a dust spectrometer (Grimm Aerosol Technik, Germany), which uses the light scattering method was used. Both systems measured the fine dust concentration every minute for 24 hours a day.
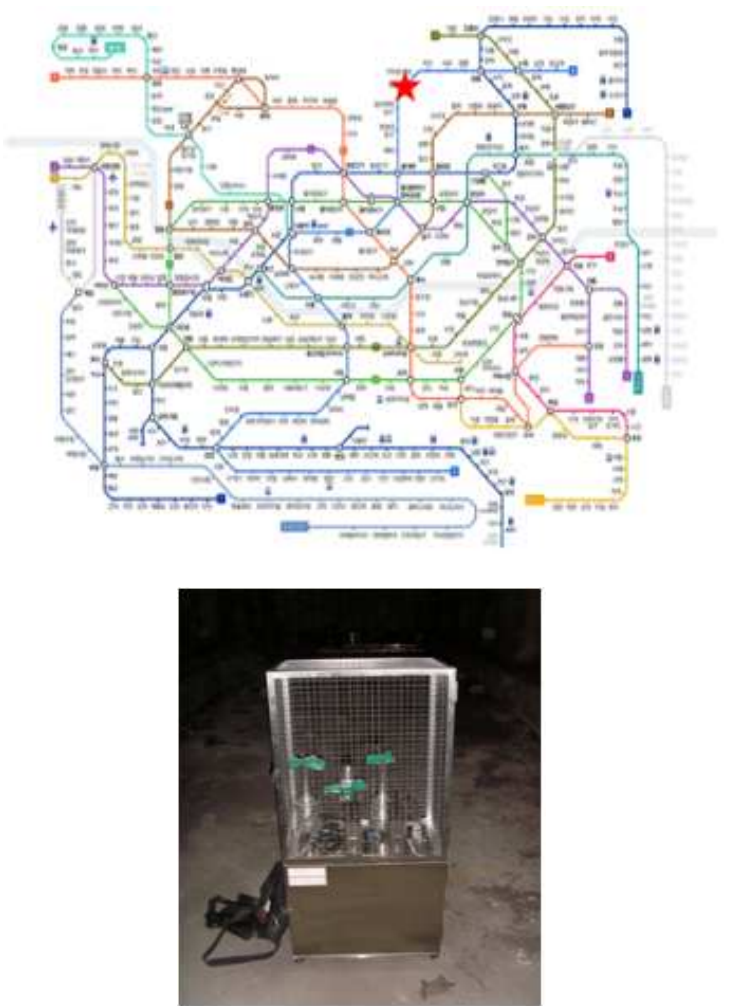

Fig. 2. Particulate matter sampling site and sampling station in the subway tunnel.

Measurement values differed between the dust spectrometer and PMX. To minimize these differences, PMX measurements were calibrated using statistical methods. For the calibration, a simple linear regression analysis and a non-linear regression analysis were used.

A regression analysis is a statistical analysis technique which identifies the correlation among variables by first assuming a mathematical model and then estimating the model from the measured data. Generally, this estimation model is used to predict or statistically deduce values. As an indication of the suitability of the estimated regression curve, the coefficient of determination was used. The coefficient of determination ranges from $0 \leq \mathrm{R}^{2} \leq 1$; the regression curve is more significant as $\mathrm{R}^{2}$ becomes closer to 1 (Seber, 1997).

Ideally, a developed fine dust measurement system would measure the same concentrations as the dust spectrometer. However, there were always differences between the initial measured values of two systems. To minimize these differences, we calibrated the PMX 
measurements using the correlation from a simple linear regression analysis. Equation 1 shows the equation of the simple linear regression:

$$
y_{i}=x_{i}^{\prime} \beta+\varepsilon_{i}
$$

In Equation 1, $x_{i}^{\prime}$ is a vector of predictors of the $i^{\text {th }}$ sample from the observed system $n ; \beta$ is a vector of the simple linear regression variables calculated with the regression analysis; and $y_{i}$ is the $i^{\text {th }}$ value of the observed system $n$ restructured by Equation 1 (Fox, 2002). In this study, the fine dust concentration was set to $x_{i}^{\prime}$ before calibration and $y_{i}$ after calibration.

If there was no linear correlation between the measured values using PMX and the dust spectrometer, the correlation was calculated using nonlinear regression, given by Equation 2, and used to calibrate the measured PMX values.

$$
y_{i}=f\left(\beta, x_{i}^{\prime}\right)+\varepsilon_{i}
$$

$\beta$ in Equation 2 is a vector of calculated nonlinear regression variables; $x_{i}^{\prime}$ is a vector of the fine dust concentrations confirmed using the observed system; and $y_{i}$ is a vector of fine dust concentrations restructured by Equation 2. Finally, $\varepsilon_{i}$ is the random error (Fox, 2002).

\section{Results and Discussion}

To calibrate PMX, the dust spectrometer (whose data were confirmed using numerous stability tests) was used. The sampling interval of the two systems was adjusted to 1 minute, and thus 1,440 data points were sampled in a 24hour period. Table 1 summarizes the 10-minute averages of the measured values of the two systems. As shown in Table 1, the concentration differences between PMX and the dust spectrometer averaged $58 \mu \mathrm{g} / \mathrm{m}^{3}$ for $\mathrm{PM}_{10}$ and $70 \mu \mathrm{g} / \mathrm{m}^{3}$ for $\mathrm{PM}_{2.5}$.

\begin{tabular}{|c|c|c|c|c|c|c|c|c|}
\hline & & \multirow{2}{*}{ Min } & \multicolumn{3}{|c|}{ Percentile } & \multirow{2}{*}{-Max } & \multirow{2}{*}{ Avg } & \multirow{2}{*}{ SD } \\
\hline & & & 25 & 50 & 75 & & & \\
\hline \multirow{2}{*}{ PMX } & $\mathrm{PM}_{10}$ & 32.9 & 73.2 & 108.4 & 124.8 & 165.5 & 98.3 & 35.9 \\
\hline & $\mathrm{PM}_{2.5}$ & 30.5 & 64.6 & 94.7 & 109.3 & 144.2 & 86.5 & 30.8 \\
\hline \multirow{2}{*}{$\begin{array}{l}\text { Dust } \\
\text { spectrometer }\end{array}$} & $\mathrm{PM}_{10}$ & 12.7 & 31.5 & 41.6 & 48.9 & 80.2 & 40.7 & 13.1 \\
\hline & $\mathrm{PM}_{2.5}$ & 4.7 & 12.8 & 18.2 & 20.6 & 27.3 & 16.6 & 5.7 \\
\hline
\end{tabular}

Table 1. Summary of the average mass concentration $\left(\mu g / \mathrm{m}^{2}\right)$.

Figure 3 shows the daily $\mathrm{PM}_{10}$ and $\mathrm{PM}_{2.5}$ concentration changes in the two systems, which show bimodal shapes. The concentration gradually decreased beginning at 1:00 AM (which is when subway operations close) and then rapidly increased beginning at 5:00 AM (when subway operation begins). This shape is similar to the results of Imre (2007) for a subway platform in Austria using a tapered element oscillating microbalance (Model 1400a, USA). Thus, this bimodal shape would likely be found in all environments which operate on a fixed schedule, such as subways.

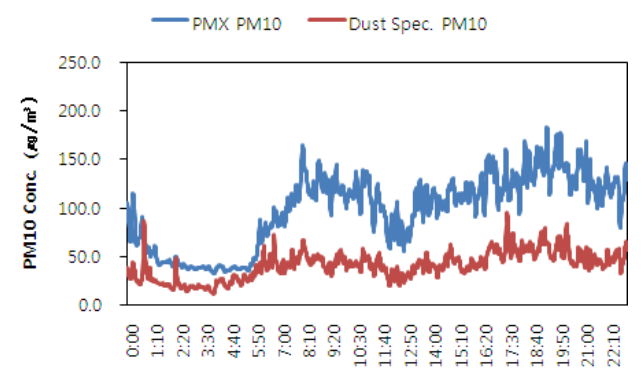

a) $\mathbf{P M}_{10}$

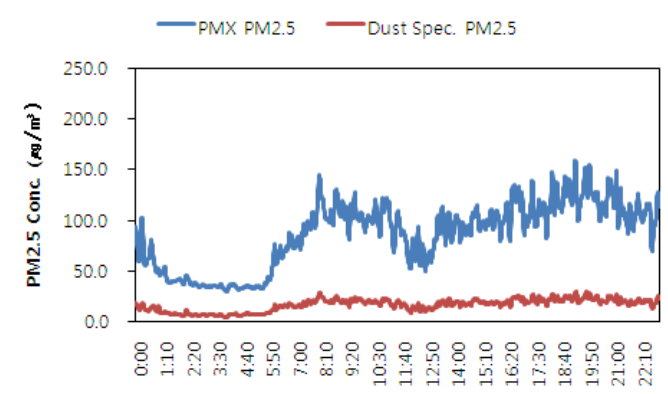

b) $\mathbf{P M}_{2.5}$

Fig. 3. Daily concentration variations of a) $P M_{10}$ and b) $P M_{2.5}$ measured by $P M X$ and the dust spectrometer.

Figure 4 is a schematization of the simple correlation analysis result. The coefficient of determination was 0.7 for $\mathrm{PM}_{10}$ and 0.9 for $\mathrm{PM}_{2.5}$. These correlation results are similar to but slightly lower than those reported by Kim et al. (2009). However, as shown in Figure 4, the data were concentrated near the linear trend curve, indicating that the measurement results of $\mathrm{PM}_{10}$ and $\mathrm{PM}_{2.5}$ were reliable.

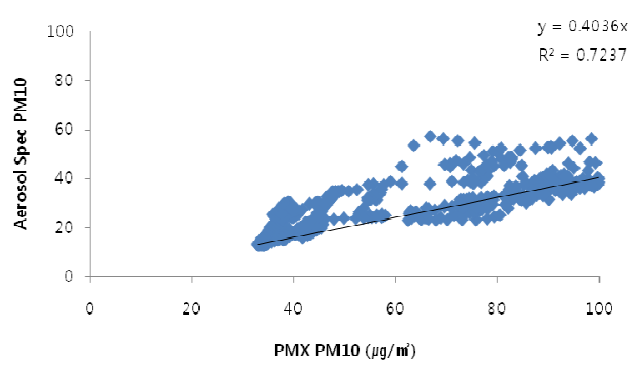

a) $\mathbf{P M}_{10}$

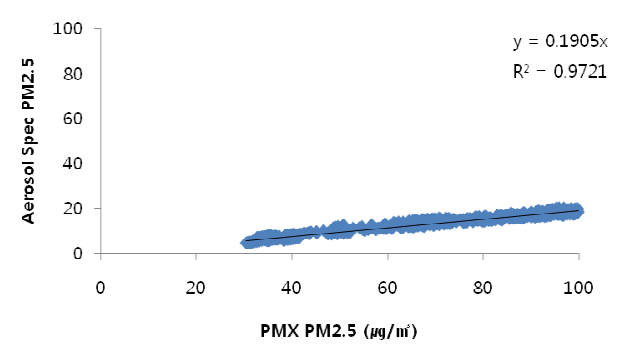

b) $\mathbf{P M}_{2.5}$

Fig. 4. Correlation coefficients of a) $P M_{10}$ and b) $P M_{2.5}$ using $P M X$ and the dust spectrometer. 


\subsection{Simple Linear Regression Analysis}

Calibration is required to use PMX as a commercial product. For statistical analysis, popular regression methods were used. A regression analysis is a statistical analysis technique used to identify correlation among variables by first assuming a mathematical model and then estimating the model from the measured data (Seber, 1997; Ahn et al., 2004). The simple linear regression model used for data calibration is shown in Equation 3 for $\mathrm{PM}_{10}$ and Equation 4 for $\mathrm{PM}_{2.5}$.

$\mathrm{PM}_{10}$,

$$
\text { Aerosol Spectrometwer }=0.4036(P M X)
$$

$\mathrm{PM}_{2.5}$,

$$
\text { Aerosol Spectrometwer }=0.1905(P M X)
$$

Table 2 summarizes the data calibrated using the simple linear regression analysis. The calibration result shows that the average PMX values were $39.7 \mu \mathrm{g} / \mathrm{m}^{3}$ for $\mathrm{PM}_{10}$ and $16.5 \mu \mathrm{g} / \mathrm{m}^{3} \quad \mathrm{PM}_{2.5}$. These values were similar to those of the dust spectrometer, which were $40.7 \mu \mathrm{g} / \mathrm{m}^{3}$ for $\mathrm{PM}_{10}$ and $16.6 \mu \mathrm{g} / \mathrm{m}^{3}$ for $\mathrm{PM}_{2.5}$. Figure 5 shows the time-series analysis after calibration. In the figure, both a) $\mathrm{PM}_{10}$ and b) $\mathrm{PM}_{2.5}$ have similar time-series graphs. The correlation coefficients from the simple linear regression were 0.7 for $\mathrm{PM}_{10}$ and 0.9 for $\mathrm{PM}_{2.5}$. As mentioned above, there was no difference in the correlation coefficients obtained by simple linear regression.

Table 2. Summary of average PM concentration using PMX and the dust spectrometer after simple linear regression $\left(\mu \mathrm{g} / \mathrm{m}^{3}\right)$.

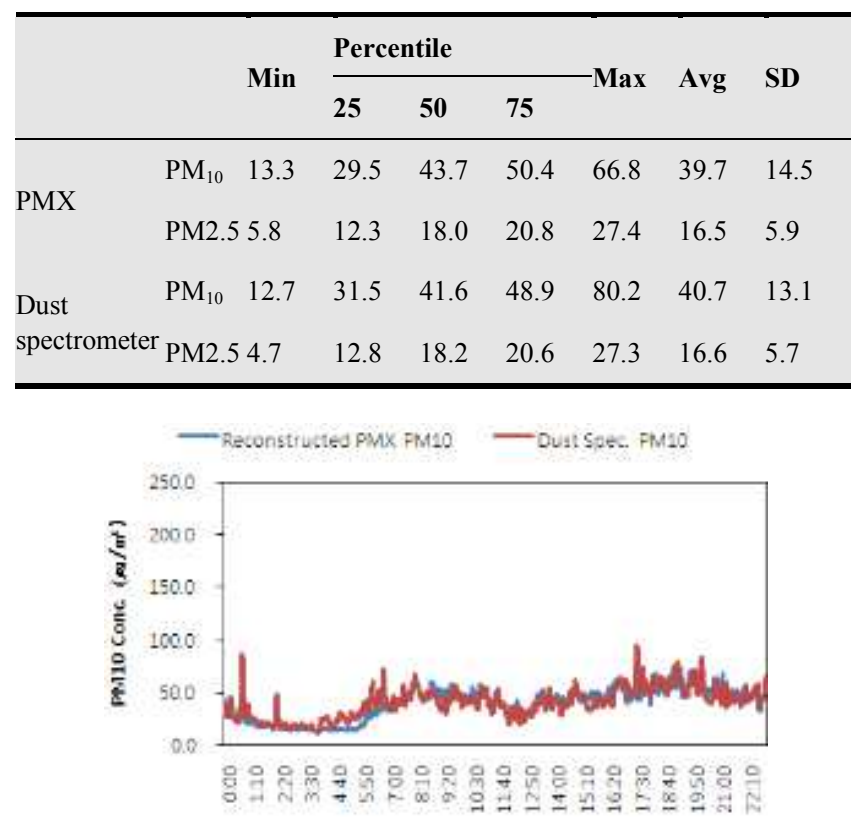

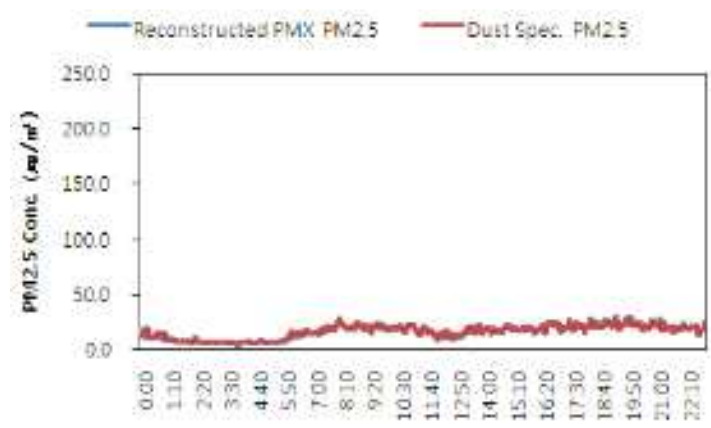

Fig. 5. Daily concentration variations of a) $P M_{10}$ and b) $P M_{2.5}$ using $P M X$ and the dust spectrometer after simple linear regression.

Kwon et al. (2009) reported coefficients of determination of $0.81 \sim 0.90$ in a study using three different $\mathrm{CO}_{2}$ measurement systems with a wireless sensor network. Our coefficients of determination showed trends similar to theirs, although the values were slightly different.

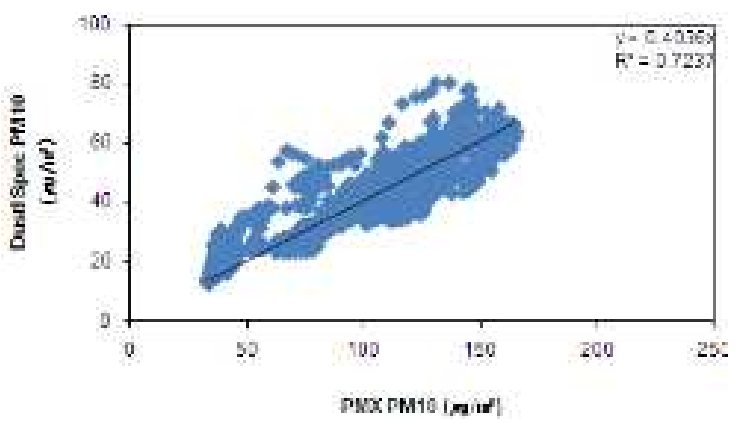

a) $\mathbf{P M}$

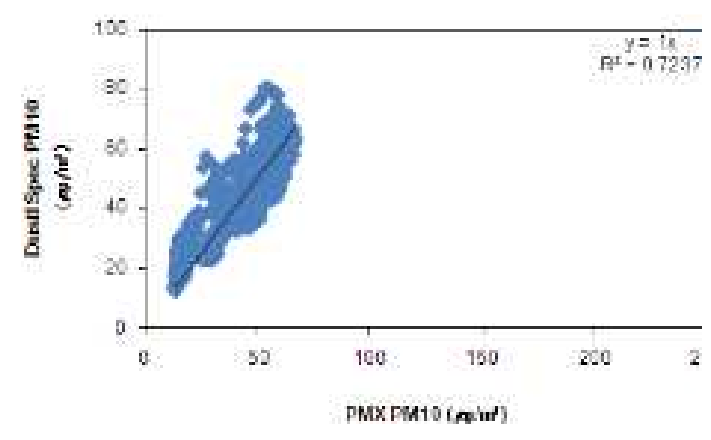

b) $\mathbf{P M}_{2.5}$

Fig. 6. Correlation coefficients of a) $P M_{10}$ and b) $P M_{2.5}$ using $P M X$ and the dust spectrometer after simple linear regression.

\subsection{Nonlinear Regression Analysis}

Although PMX was calibrated using simple linear regression analysis, the correlation coefficients were somewhat low at 0.7 for $\mathrm{PM}_{10}$ and 0.9 for $\mathrm{PM}_{2.5}$. Therefore, a nonlinear regression method was performed on the models in Equations 5 and 6 to improve the reliability of PMX.

$\mathrm{PM}_{10}$

$$
\text { Aerosol Spectrometwer }=1.469(P M X)^{0.724}
$$




$$
\begin{aligned}
& \mathrm{PM}_{2.5} \text {, } \\
& \text { Aerosol Spectrometwer }=0.2237(P M X)^{0.9556}
\end{aligned}
$$

Table 3 summarizes the PMX data calibrated with nonlinear regression. The PMX averages were $40.2 \mu \mathrm{g} / \mathrm{m}^{3}$ for $\mathrm{PM}_{10}$ and $16.6 \mu \mathrm{g} / \mathrm{m}^{3}$ for $\mathrm{PM}_{2.5}$. The PM averages by nonlinear regression of PMX were close to the values measured by the dust spectrometer of $40.7 \mu \mathrm{g} / \mathrm{m}^{3}$ for $\mathrm{PM}_{10}$ and $16.6 \mu \mathrm{g} / \mathrm{m}^{3}$ for $\mathrm{PM}_{2.5}$. The standard deviation of the linear regression analysis was even more similar to the measured values using the dust spectrometer.

Table 3. Summary of average PM concentration using PMX and the dust

\begin{tabular}{|c|c|c|c|c|c|c|c|c|}
\hline & & \multirow{2}{*}{ Min } & \multicolumn{3}{|c|}{ Percentile } & \multirow{2}{*}{-Max } & \multirow{2}{*}{ Avg } & \multirow{2}{*}{ SD } \\
\hline & & & $\overline{25}$ & 50 & 75 & & & \\
\hline \multirow{2}{*}{ PMX } & $\mathrm{PM}_{10}$ & 18.5 & 33.0 & 43.9 & 48.6 & 50.9 & 40.2 & 11.3 \\
\hline & $\mathrm{PM}_{2.5}$ & 6.1 & 12.5 & 18.1 & 20.8 & 27.2 & 16.6 & 5.7 \\
\hline \multirow{2}{*}{$\begin{array}{l}\text { Dust } \\
\text { spectromete }\end{array}$} & $\mathrm{PM}_{10}$ & 12.7 & 31.5 & 41.6 & 48.9 & 80.2 & 40.7 & 13.1 \\
\hline & $\mathrm{rM}_{2.5}$ & 4.7 & 12.8 & 18.2 & 20.6 & 27.3 & 16.6 & 5.7 \\
\hline
\end{tabular}
spectrometer after nonlinear regression $\left(\mu \mathrm{g} / \mathrm{m}^{s}\right)$.
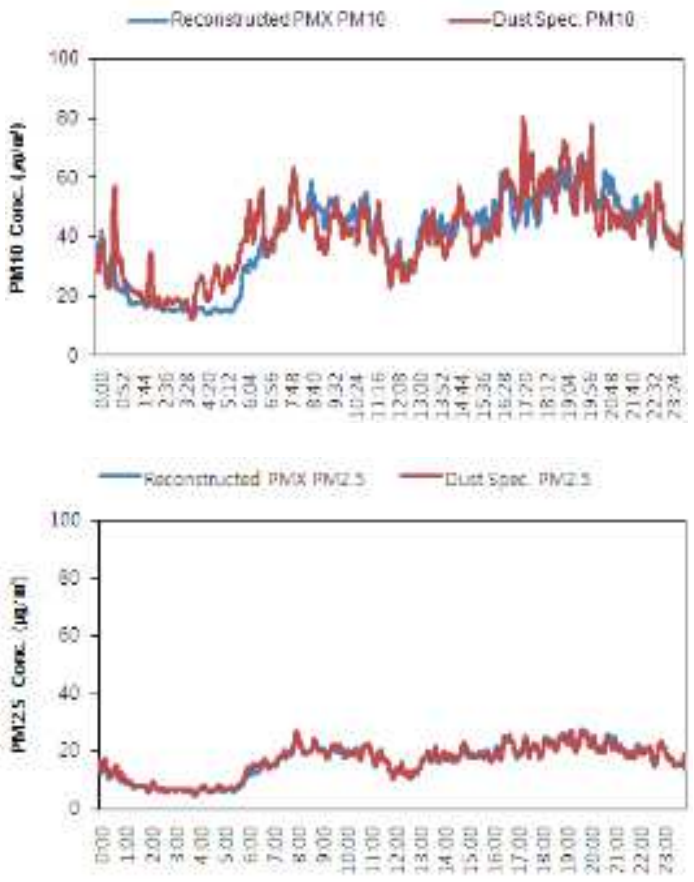

Fig. 7. Daily concentration variations of a) $P M_{10}$ and b) $P M_{2.5}$ using $P M X$ and the dust spectrometer after nonlinear regression.

The non-linear regression analysis obtained correlation coefficients of 0.8 for $\mathrm{PM}_{10}$ and 0.9 for $\mathrm{PM}_{2.5}$. The correlation coefficient of the nonlinear regression was higher for $\mathrm{PM}_{10}$ than for the linear regression, while the two methods were similar for $\mathrm{PM}_{2.5}$. Kim et al. (2009) also found higher correlation coefficients by nonlinear than by linear regression in their study using the light scattering and beta ray absorption methods for $\mathrm{PM}_{10}$.
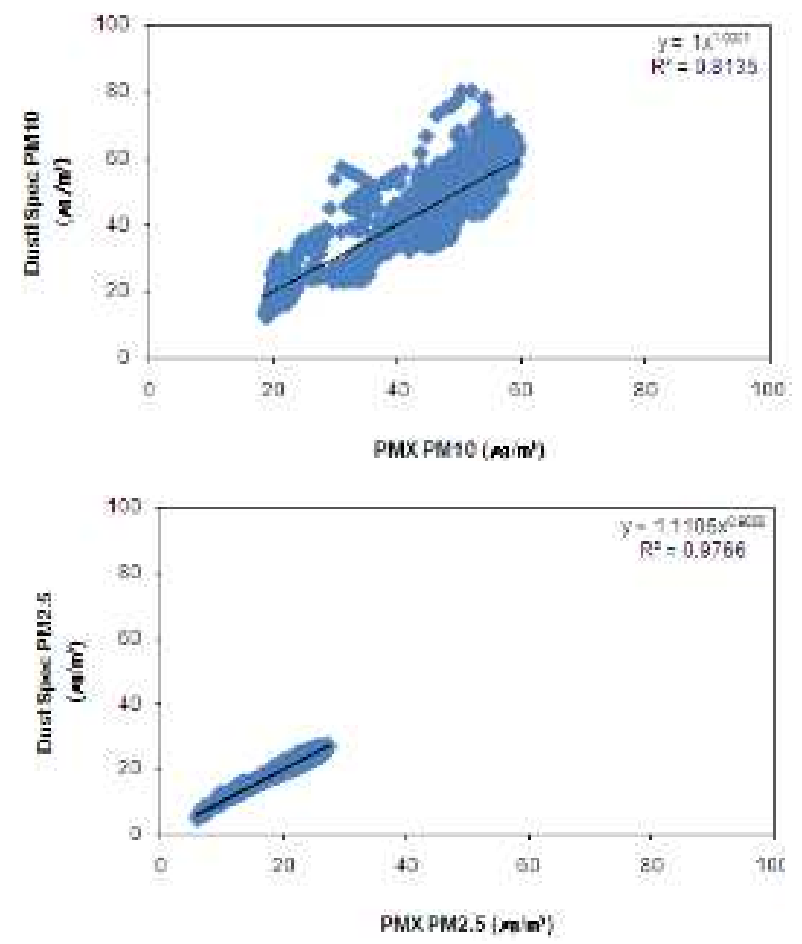

Fig. 8. Correlation coefficients of a) $P M_{10}$ and b) $P M_{2.5}$ using $P M X$ and a dust spectrometer after nonlinear regression.

\section{Conclusions}

Korea's Ministry of the Environment regulates subway operations and has prepared a long-term plan and invested a huge budget into reducing fine dust concentrations in subway stations. However, these efforts have not yet produced results. Ventilation is the most common way to reduce fine dust concentrations, but the cost of fan operation places a heavy burden on operating costs. Since HVAC systems dedicated to fine dust concentrations are not yet common, it would be very useful to develop an accurate and low-cost fine dust measurement system. In this study, PMX was developed as a fine dust measurement system. To test the reliability of PMX, the system was installed at the same location as a dust spectrometer (which has been evaluated in several previous studies) at Gireum Station in the Seoul Metropolitan Subway on Line No. 4.

The two systems showed a similar time-series trend, although the measured values were somewhat different. A simple correlation analysis of the two data groups showed coefficients of determination of 0.7 for $\mathrm{PM}_{10}$ and 0.9 for $\mathrm{PM}_{2.5}$. For the PMX data, most data were concentrated around the trend curve. Therefore, calibration of PMX data was required prior to application in the field. For the calibration, simple linear regression and nonlinear regression were applied. The correlation coefficients of simple linear regression were 0.8 for $\mathrm{PM}_{10}$ and 0.9 for $\mathrm{PM}_{2.5}$, while those for nonlinear regression were 0.7 for $\mathrm{PM}_{10}$ and 0.9 for $\mathrm{PM}_{2.5}$. For $\mathrm{PM}_{10}$, the nonlinear regression showed a higher correlation coefficient, suggesting that nonlinear regression is preferable to linear regression for calibrating the PMX system. 
PMX can monitor the air quality in real time in large spaces, such as a subway station, at a lower cost than existing commercial products. It can increase the efficiency of the subway ventilation system and reduce power consumption by analyzing the air quality status in real-time and interfacing with the ventilations system. However, the developed system requires calibration before it can be applied in the field, as shown in this study. Therefore, further studies are required to observe the air quality sensors and ensure high reliability and durability.

\section{References}

[1] Ahn, Y.J., Kwon, W.T., Kim, Y.W., 2004. Estimation of tool life by simple \& multiple linear regression analysis of Si3N4 ceramic cutting tools, Transaction of the Korean Society of Machine Tool Engineers, 13(4), 23-29.

[2] Chow, J.C., Watson, J.G., 1998. Guideline on speciated particulate monitoring, Office of Air Quality Planning and Standards U.S. EPA, NC 27711.

[3] Coffey, C.C., Pearce, T.A., 2010. Direct-reading methods for workplace air monitoring, J. of Chemical Health \& Safety, 17(3), 10-21.

[4] Dockery, D.W., Pope, C.A., Xu, X.P., Spengler, J.D., Ware, J.H., Fay, M.E., Ferris, B., Speizer, F.E., 1993. An association between air-pollution and mortality in 6 United States cities, New England J. of Medicine, 329, 1753-1759.

[5] Fox, J., 2002. Nonlinear regression and nonlinear least squares, Appendix to an $\mathrm{R}$ and S-PLUS companion to applied regression.

[6] Imre S., Weidinger, T., Maenhaut W., 2007. Time-resolved mass concentration, composition and sources of aerosol particles in a metropolitan underground railway station, Atmospheric Environment, 41(37), 8391-8405.

[7] Jung, C.H., Cho, Y.S., Hwang, S.M., Jung, Y.G., Ryu, J.C., Shin, D.S., 2007. Analysis of measurement error for PM10 mass concentration by inter-comparison study, J. KOSAE, 23(6), 689-698.

[8] Kim, S.J., Son, Y.S., Kang, H.S., Kim, J.C., Lee, J.H., Kim, G.S., Kim, I.W., 2009 Proceeding of the 49th Meeting of KOSAE, 613-615.

[9] Kwon, J.W., Kim, J.C., Kim, G.S., 2009. Air quality monitoring system using NDIR-CO2 sensor for underground space based in wireless sensor network, J. of the IEEK, 46(4), 23-38.

[10] Pope, C.A., Bates, D.V., Raizenne, M.E., 1995. Health effects of particulate air pollution: time for reassessment, Environmental Health Perspectives, 103, 472-480.

[11] Seber, G.A.F., 1997. Linear regression analysis, John Wiley \& Sons, New York. 